\title{
Pancreatic cancer incidence trends in the United States from
}

\section{0-2017: Analysis of Surveillance, Epidemiology and End}

\section{Results (SEER) database [version 1; peer review: 2 approved]}

\author{
Hassam Ali (D1), Rahul Pamarthy1, Meghana Vallabhaneni1, Shiza Sarfraz (D)2, \\ Hadiqa Ali², Hamza Rafique ${ }^{2}$ \\ ${ }^{1}$ Internal Medicine, East Carolina University, Greenville, North Carolina, 27834, USA \\ 2Internal Medicine, University of Health Sciences, Lahore, Punjab, 42000, Pakistan
}

\author{
V1 First published: 02 Jul 2021, 10:529 \\ https://doi.org/10.12688/f1000research.54390.1 \\ Latest published: $02 \mathrm{Jul}$ 2021, 10:529 \\ https://doi.org/10.12688/f1000research.54390.1
}

\section{Abstract}

Background: Recent incidence trends of pancreatic cancers were reviewed by demographics and histologic type to observe any new findings.

Methods: Data was used from the Surveillance, Epidemiology, and End Results (SEER) registry 18 (2000-2017) and it underwent temporal trend analysis. Pancreatic cancer incidence rates were reported based on histological subtype and demographics.

Results: The incidence rate of white males increased significantly during 2000-2017 (annual percent change (APC) $=3.5 \%$ ) compared to previously reported APCs. The incidence of white females grew from an APC of $1.29 \%$ to $2.9 \%$. Rates among black ethnicity increased with an APC of $4.2 \%$. Rates among Hispanics and other ethnicities also showed increment. The rates for ductal adenocarcinoma showed a positive trend in all races, with the APC $\geq 6 \%$ for females and APC $\geq$ $6.5 \%$ for males. The rates of non-secretory endocrine tumors showed a decline in both genders of all five races in recent years after showing an initial positive trend till 2010. Rates for pancreatic adenocarcinoma continued to rise in all ethnicities from 2000-2017. Interestingly, there was a rise in carcinoid type pancreatic neuroendocrine tumors (PNETs) in all ethnicities. Cumulatively, males had a higher incidence than females; male to female Incidence Risk Ratio (IRRs) was 1.32. The IRR was $>1$ for age groups $\geq 35$ years. The male to female IRRs was less than 1 for cystic adenocarcinoma, secretory endocrine, and solid pseudopapillary carcinomas (IRR $=0.5,0.9$, and 0.2 respectively, confidence intervals $0.4-0.6$ and $0.9-1.3,0.2-0.3$, respectively). Conclusion: Pancreatic cancer incidence continued to rise in the years 2000-2017. However, incidence differed by demographics and histologic type. Interestingly, recent years discerned a rise in PNETs (carcinoid type) which has not been reported previously.

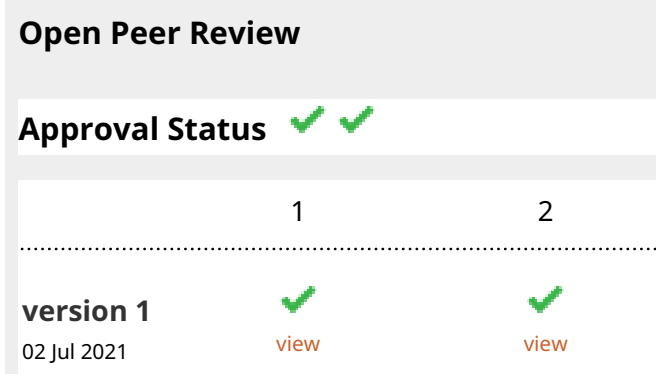

1. Syed Hamza Bin Waqar (D), Downstate Health Sciences University, Brooklyn, USA

2. Harigopal Sandhyavenu, Mayo Clinic, Rochester, USA

Any reports and responses or comments on the article can be found at the end of the article. 


\section{Keywords}

Pancreatic cancer, SEER database, PNETs, pancreatic neuroendocrine tumors, pancreatic cancer risk factors, Pancreatic cancer incidence, SEER 18

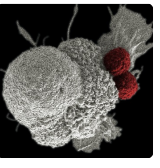

This article is included in the Oncology

gateway.

Corresponding author: Hassam Ali (alih20@ecu.edu)

Author roles: Ali H: Conceptualization, Methodology, Software, Supervision, Writing - Original Draft Preparation, Writing - Review \& Editing; Pamarthy R: Investigation, Project Administration, Visualization; Vallabhaneni M: Data Curation, Writing - Original Draft Preparation; Sarfraz S: Resources, Software, Supervision; Ali H: Software, Validation; Rafique H: Writing - Review \& Editing

Competing interests: No competing interests were disclosed.

Grant information: The author(s) declared that no grants were involved in supporting this work.

Copyright: $\odot 2021 \mathrm{Ali} \mathrm{H}$ et al. This is an open access article distributed under the terms of the Creative Commons Attribution License, which permits unrestricted use, distribution, and reproduction in any medium, provided the original work is properly cited.

How to cite this article: Ali $\mathrm{H}$, Pamarthy R, Vallabhaneni $\mathrm{M}$ et al. Pancreatic cancer incidence trends in the United States from 20002017: Analysis of Surveillance, Epidemiology and End Results (SEER) database [version 1; peer review: 2 approved] F1000Research 2021, 10:529 https://doi.org/10.12688/f1000research.54390.1

First published: 02 Jul 2021, 10:529 https://doi.org/10.12688/f1000research.54390.1 


\section{Introduction}

As per global reports in 2018, pancreatic cancer remains the twelfth most common cancer in men and the eleventh in women $^{1}$. Cancer-related deaths have pancreatic cancer as the seventh leading cause ${ }^{1}$. In the United States of America (USA), pancreatic cancer (PC) incidence has been increasing as previously reported ${ }^{1,2}$. PC mortality is estimated to rise as time progresses, and recent projections estimate that it might overpass colorectal cancer to become the second leading cancer-related cause of death after lung cancer ${ }^{3}$. In 2020 reports showed 57,600 new cases and 47,050 PC related deaths ${ }^{3}$. The PC risk increases with age, and the median age of diagnosis is 71 years ${ }^{4}$. Previously the increases were most significant for non-secretory endocrine cancers, followed by ductal adenocarcinomas and adenocarcinoma 5 .

\section{Methods}

The results were obtained from the analysis of the Surveillance Epidemiology and End Results (SEER) cancer registry database. SEER 18 includes $28 \%$ of the U.S. population as of the 2010 census $^{6}$. The rates are available by expanded race/ethnicity of cases diagnosed, including white, black, Asian/Pacific Islander, and American Indian/Alaskan Native and Hispanic ethnicity. SEER 18 also includes adjustments for areas impacted by hurricanes Katrina and Rita. It contains a record for each of 8,131,919 tumors. 19 age groups are available and can be classified as < 1 year, 1-4 years, 5-9 years to $85+$ years, or unknown. The registries included in SEER 18 are San FranciscoOakland SMSA, Connecticut, Detroit (Metropolitan), Hawaii, Iowa, New Mexico, Seattle (Puget Sound), Utah, Atlanta (Metropolitan, San Jose-Monterey, Los Angeles, Alaska Natives, Rural Georgia, California excluding San Francisco, San Jose Monterey, Los Angeles, Kentucky, Louisiana, New Jersey, Greater Georgia. SEER 18 encompasses the SEER 13, which itself includes seer 9 in addition to supplementary registries ${ }^{6}$.

Within the SEER database, International Classification of Diseases (ICD)-0-2 was used through $2000^{7}$, and ICD-0-3 following 2001 for coding the primary site and histologic type of diagnosed pancreatic cancer cases $^{8}$. Site recode ICD-0-3/WHO 2008: Pancreas (ICD-O-3 site C25) was used via SEER*Stat software to select cases pancreatic cancer'. Only cases that were malignant and had a microscopically confirmed diagnosis were included. The ages below 30 years were filtered out due to reportedly low incidence per previous literature ${ }^{5}$. Cases were characterized into exclusive groups based on morphological classification of pancreatic cancer in the ICD (Numbers given below) for Oncology and previous similar study ${ }^{5,8}$. In addition to the undermentioned cases, all other cases were classified as poorly specified types.

- $\quad$ Adenocarcinoma, not otherwise specified (NOS) (8140)

- $\quad$ Mucinous adenocarcinoma $(8480,8481)$

- Ductal adenocarcinoma excluding cystic or mucinous $(8507,8510,8514,8521,8560,8570,8523,8255$, $8490,8500)$

- $\quad$ Cystic adenocarcinoma $(8440,8470,8504)$
- Intraductal papillary mucinous neoplasm (IPMN) $(8453,8471,8503,8144,8450)$

- $\quad$ Non-secretory endocrine $(8150,8246)$

- $\quad$ Secretory endocrine $(8151,8152,8156,8153,8155)$

- $\quad$ Carcinoid tumors $(8243,8244,8245,8240,8241)$

- Acinar cell adenocarcinomas $(8550,8551)$,

- $\quad$ Solid pseudopapillary tumors (8452),

- Other adenocarcinoma (8145, 8260, 8441, 8154, $8574,8460)$

- $\quad$ Non-carcinomas (8680-9999)

SEER* Stat $^{9}$ was utilized to calculate the incidence and ageadjusted rates, grouped by histologic subtype, gender, ethnicity, and age group. SEER 18 registry was used for analyzing tends with detailed racial group categories from 2000 to 2017 using yearly diagnosis. The period-specific rates were plotted and utilized temporal trend figures at two-year intervals and utilized a logarithmic scale with a base of 10 degrees portrayed rate per 100,000 person-years ${ }^{10}$. The annual percent changes (APCs) were quantified using annual rates for temporal trends (Table 1 \& Table 2), and 95\% confidence intervals (CIs) were included. SEER*Stat ${ }^{9}$ was used for all analyses ${ }^{6}$. Incidence Rate Ratios (IRRs) based on gender and ethnicity/race were calculated; these were further classified based on age groups and histological types. 95\% CIs for the IRRs was calculated using the Tiwari method, and the figures were generated using Microsoft excel. Potential sources of bias include concern of patient migration across seer registries. Data is de-identified by SEER, and this potential for sample bias cannot be intervened but mentioned in this study's limitations.

\section{Results}

Temporal trends

SEER 18 allowed us to explore short-term, nonetheless, relatively recent and previously unreported trends in pancreatic cancer (Table 1) (Figure 1). The incidence rate of white males has increased significantly during 2000-2017 (APC = $3.5 \%$ ) compared to previously reported APC of $0.95 \%$ from 1994-2013 ${ }^{5}$. The incidence of white females also increased from an APC of $1.29 \%(1999-2013)$ to $2.9 \%(2000-2017)^{5}$. Rates among black males and females have also increased in recent years, per our analysis, with an APC of $4.2 \%$ each, respectively. Previously, the rates among black males decreased, while rates remained unchanged among black females (1975-2013) ${ }^{5}$.

Rates among Hispanic men and women have continued to increase $(\mathrm{APC}=6.5 \%$ and $6.4 \%)$. Similarly, rates among American Indian/Alaska Native in men increased more than women (APC $=7.4 \%$ vs. $2.1 \%$ ). In Asian or Pacific Islander, the APC was similar in both genders $(6.1 \%)$ as compared to lower incidence rates of $0.16 \%$ for males and $1.23 \%$ for females $(1992-2013)^{5}$.

There was a positive trend in rates for white males from ages 30-39 and 50 years for the 5-year age groups up to 85 and 
Table 1. Total cases and Annual Percentage Changes (APCs) of pancreatic cancer by race and gender (2000-2017).

\begin{tabular}{|c|c|c|c|c|}
\hline \multicolumn{5}{|c|}{ Males } \\
\hline Race & Count & APC & LCI & UCI \\
\hline White & 56991 & $3.4^{*}$ & 3.3 & 3.6 \\
\hline Black & 8807 & $4.2^{*}$ & 3.8 & 4.6 \\
\hline American Indian/Alaska Native & 396 & $7.4^{*}$ & 4.7 & 10.1 \\
\hline Asian or Pacific Islander & 5138 & $6.1^{*}$ & 5.6 & 6.7 \\
\hline Hispanic (All Races) & 7616 & $6.5^{\star}$ & 6 & 7 \\
\hline Non-Hispanic unknown races & 133 & $17.1^{*}$ & 12.2 & 22.2 \\
\hline \multicolumn{5}{|c|}{ Females } \\
\hline Race & Count & APC & LCI & UCI \\
\hline White & 50344 & $2.9^{*}$ & 2.7 & 3 \\
\hline Black & 9489 & $4.2^{*}$ & 3.8 & 4.6 \\
\hline American Indian/Alaska Native & 383 & $2.7^{\star}$ & 0.7 & 4.7 \\
\hline Asian or Pacific Islander & 5404 & $6.1^{*}$ & 5.6 & 6.7 \\
\hline Hispanic (All Races) & 7751 & $6.4^{*}$ & 5.8 & 7 \\
\hline Non-Hispanic unknown races & $\sim$ & $\sim$ & $\sim$ & $\sim$ \\
\hline
\end{tabular}

APC=Annual percent change; $U C I, L C I=U p p e r$, Lower 95\% confidence interval.

Confidence intervals are 95\% for trends.

Percent changes were calculated using 1 year for each end point; APCs were calculated using weighted least squares method.

Statistic could not be calculated due to lower number of cases or at least one year with no reported cases.

* The APC is significantly different from zero $(p<0.05)$.

Cases and annual percentage changes were only mentioned if there were enough cases to yield results after temporal trends analysis.

above as shown in Table 2 and Figure 2. White females showed a statistically significant positive trend from age $\geq 50$. Interestingly, ages 40-49 showed a negative trend in white males, and age group 40-44 had a statistically significant negative APC of $-2.3 \%$.

Both genders in the black population showed a positive trend after age $\geq 45$ (Table 2) (Figure 2). It was impossible to calculate APCs among younger age groups for black females due to at least one year with zero cases. Age-specific rates also rose among Asians or pacific islanders and Hispanic men from ages $\geq 35$ and $\geq 40$, respectively (Figure 2). APCs for females of Asians or pacific islanders and Hispanic ethnicity also showed a positive trend after ages $\geq 40$ and $\geq 35$, respectively (Figure 2). Further analysis was performed to explore trends in American Indian/Alaskan native (Figure 2). However, APCs could not be calculated in either males or females for any age group due to at least one year with zero cases. The results are therefore not shown in tables. From 2000 to 2017, for ages, more than
30 years, 152,548 of 190,586 pancreatic cancer cases $(80.04 \%)$ diagnosed had a microscopic confirmation. This represented an increase compared with the $77 \%$ microscopically confirmed cases from 1992-2013, including all ages. (Results are not shown). We did not report the analysis of the temporal trends by following histologic-type groups due to the number of cases being too low: cystic adenocarcinoma $(n=408)$, ductal specified as arising from an IPMN $(n=520)$, secretory endocrine $(n=203)$, other adenocarcinomas: acinar cell $(n=438)$, solid pseudopapillary tumors $(n=228)$, other adenocarcinomas $(n=448)$, other non-carcinomas $(n=6)$.

Rates for pancreatic adenocarcinoma, the most common histologic type of pancreatic cancer, continued to rise in all ethnicities from 2000-2017. The rates for ductal adenocarcinoma (excluding mucinous and cystic) showed a positive trend in all races (including American Indian/Alaska Native) with the APC $\geq 6 \%$ for females and APC $\geq 6.5 \%$ for males (Table 2) (Figure 3). The rates of non-secretory endocrine tumors showed a decline in both genders of all five races in recent years after showing an initial positive trend till 2010. However, the APC from 20002017 remained positive and $\geq 3.5 \%$ for males and females (Table 2). APC for American Indian/Alaska Native was not calculated due to at least one year with zero cases; however, trends are reported in Figure 3.

Interestingly, the rates for carcinoid endocrine tumors showed a positive trend and rise in rates from 2010 and onwards (Figure 3), which was not reported previously. The APC was highest in white males and females, and black females $(32.5 \%$, $30.3 \%$, and $26.9 \%$, respectively). APCs for the rest of the races were not reported as statistics were incalculable as at least one year had no reported cases from 2000 to 2017.

\section{Male to female IRRs}

In SEER 18 (2000-2017), 152,548 cases were microscopically confirmed from ages $\geq 30$; males: 79081 and females: 73,467. This was higher than previously reported rates, which was 69,049 in males and 69,548 in females $(2000-2013)^{5}$. The IRR was greater in males' total cases (IRR 1.32,95\% CI 1.30-1.33) (Table 3). All age groups from 35 and above showed an increased incidence risk in males vs. females (IRRs $>1$ : increased risk) (Table 3). Rates among males were higher compared to females for all histological subtypes except ductal cystic adenocarcinoma $(0.5,95 \%$ CI $0.4-0.6)$, secretory endocrine tumors $(0.9$, $95 \%$ CI $0.9-1.3)$, solid pseudopapillary $(0.2,95 \%$ CI $0.2-0.3)$ and non-carcinomas $(0.1,95 \%$ CI 0.7-7.7). Carcinoid endocrine tumors also showed a greater IRR in males $(1.4,95 \%$ CI 1.3-1.5) (Table 3).

\section{Incidence risk ratios IRRs by racial/ethnic group}

For SEER 18 (2000-17), the highest rates for pancreatic cancer in ages greater than 30 , were in both black males and females $($ IRR $=1.21$ and 1.36, respectively) when compared to white (non-Hispanic) ethnicity. Incidence, when compared to white (non-Hispanic) ethnicity, was higher in both black males and females (IRR $=1.21$ and 1.36, respectively). The rates for both genders in American Indian/Alaska, Native Asian, or Pacific 
Table 2. Total cases and Annual Percentage Changes (APCs) of pancreatic cancer by race, gender, and age group (2000-2017).

\begin{tabular}{|c|c|c|c|c|}
\hline \multicolumn{5}{|c|}{ Males } \\
\hline Age groups & Count & APC & LCI & UCI \\
\hline \multicolumn{5}{|l|}{ White } \\
\hline 30-34 years & 112 & $3.3^{*}$ & 0.2 & 6.4 \\
\hline 35-39 years & 359 & 0.2 & -2.1 & 2.5 \\
\hline 40-44 years & 869 & $-2.3^{*}$ & -3.7 & -0.9 \\
\hline 45-49 years & 1,989 & -0.7 & -1.5 & 0.2 \\
\hline 50-54 years & 3,931 & $0.9^{*}$ & 0.2 & 1.6 \\
\hline 55-59 years & 6,201 & $3.1^{*}$ & 2.3 & 4 \\
\hline 60-64 years & 7,997 & $4.7^{*}$ & 4 & 5.4 \\
\hline 65-69 years & 9,271 & $5.7^{*}$ & 5.1 & 6.4 \\
\hline 70-74 years & 8,978 & $3.8^{*}$ & 3.1 & 4.5 \\
\hline 75-79 years & 7,959 & $2.4^{*}$ & 1.8 & 2.9 \\
\hline 80-84 years & 5,871 & $2.7^{*}$ & 1.9 & 3.4 \\
\hline 85+ years & 3,454 & $5.4^{*}$ & 4.4 & 6.4 \\
\hline \multicolumn{5}{|l|}{ Black } \\
\hline 30-34 years & $\sim$ & $\sim$ & $\sim$ & $\sim$ \\
\hline 35-39 years & 84 & -1.3 & -5.4 & 3 \\
\hline 40-44 years & 199 & -0.5 & -3 & 2 \\
\hline 45-49 years & 484 & 0.3 & -1.6 & 2.3 \\
\hline 50-54 years & 945 & $2.0^{*}$ & 0.1 & 3.9 \\
\hline 55-59 years & 1,333 & $4.4^{*}$ & 2.9 & 6 \\
\hline 60-64 years & 1,534 & $7.0 *$ & 5.8 & 8.3 \\
\hline 65-69 years & 1,384 & $4.9^{*}$ & 3.6 & 6.2 \\
\hline 70-74 years & 1,180 & $4.3^{*}$ & 3.1 & 5.5 \\
\hline 75-79 years & 847 & $4.0^{*}$ & 2.2 & 5.9 \\
\hline $80-84$ years & 505 & $2.8^{*}$ & 1.3 & 4.4 \\
\hline $85+$ years & 264 & $7.5^{*}$ & 4.9 & 10.3 \\
\hline \multicolumn{5}{|c|}{ Asian or Pacific Islander } \\
\hline 30-34 years & $\sim$ & $\sim$ & $\sim$ & $\sim$ \\
\hline 35-39 years & $\sim$ & $\sim$ & $\sim$ & $\sim$ \\
\hline 40-44 years & 125 & 2.2 & -1.8 & 6.4 \\
\hline 45-49 years & 221 & $5.3^{*}$ & 1.8 & 9 \\
\hline 50-54 years & 353 & $6.0^{*}$ & 3.4 & 8.6 \\
\hline $55-59$ years & 513 & $4.6^{*}$ & 3.1 & 6.1 \\
\hline 60-64 years & 699 & $5.7^{*}$ & 4.3 & 7.1 \\
\hline $65-69$ years & 788 & $6.9 *$ & 5.3 & 8.5 \\
\hline
\end{tabular}




\begin{tabular}{|c|c|c|c|c|}
\hline \multicolumn{5}{|c|}{ Males } \\
\hline Age groups & Count & APC & LCI & UCI \\
\hline \multicolumn{5}{|c|}{ Asian or Pacific Islander } \\
\hline 70-74 years & 768 & $6.4^{*}$ & 5.1 & 7.7 \\
\hline 75-79 years & 690 & $5.8^{*}$ & 4 & 7.7 \\
\hline 80-84 years & 523 & $6.3^{*}$ & 4.5 & 8.3 \\
\hline $85+$ years & 353 & $7.5^{\star}$ & 5.2 & 9.9 \\
\hline \multicolumn{5}{|l|}{ Hispanics } \\
\hline 30-34 years & $\sim$ & $\sim$ & $\sim$ & $\sim$ \\
\hline 35-39 years & 123 & 1.9 & -2.4 & 6.4 \\
\hline 40-44 years & 252 & $5.7^{*}$ & 2.8 & 8.6 \\
\hline $45-49$ years & 454 & $3.9 *$ & 1.5 & 6.4 \\
\hline $50-54$ years & 745 & $6.3^{*}$ & 5 & 7.6 \\
\hline $55-59$ years & 914 & $7.2^{*}$ & 5.6 & 8.8 \\
\hline 60-64 years & 1,152 & $7.9 *$ & 6.6 & 9.2 \\
\hline $65-69$ years & 1,119 & $7.3^{*}$ & 6 & 8.6 \\
\hline 70-74 years & 1,090 & $5.5^{*}$ & 4.1 & 6.9 \\
\hline $75-79$ years & 866 & $5.3^{*}$ & 3.9 & 6.7 \\
\hline 80-84 years & 545 & $6.3^{*}$ & 3.9 & 8.8 \\
\hline $85+$ years & 307 & $8.2^{*}$ & 4.6 & 11.8 \\
\hline
\end{tabular}

\begin{tabular}{|c|c|c|c|c|}
\hline \multicolumn{5}{|c|}{ Females } \\
\hline \multicolumn{5}{|l|}{ White } \\
\hline 30-34 years & 138 & 0.8 & -2.6 & 4.3 \\
\hline 35-39 years & 284 & 0.3 & -2.4 & 3.1 \\
\hline 40-44 years & 672 & 0 & -1.6 & 1.6 \\
\hline 45-49 years & 1,427 & -0.2 & -1.6 & 1.3 \\
\hline 50-54 years & 2,697 & $1.6^{*}$ & 0.6 & 2.6 \\
\hline 55-59 years & 4,402 & $3.6^{*}$ & 2.6 & 4.6 \\
\hline $60-64$ years & 5,793 & $4.7^{\star}$ & 4.1 & 5.4 \\
\hline 65-69 years & 7,150 & $5.0^{*}$ & 4.3 & 5.8 \\
\hline 70-74 years & 7,806 & $2.5^{\star}$ & 1.7 & 3.2 \\
\hline $75-79$ years & 8,183 & $1.3^{*}$ & 0.8 & 1.7 \\
\hline 80-84 years & 6,672 & $1.7^{\star}$ & 0.9 & 2.5 \\
\hline $85+$ years & 5,120 & $4.2^{*}$ & 3.6 & 4.8 \\
\hline \multicolumn{5}{|l|}{ Black } \\
\hline 30-34 years & $\sim$ & $\sim$ & $\sim$ & $\sim$ \\
\hline 35-39 years & $\sim$ & $\sim$ & $\sim$ & $\sim$ \\
\hline 40-44 years & 194 & -0.2 & -3.3 & 3.1 \\
\hline
\end{tabular}




\begin{tabular}{|c|c|c|c|c|}
\hline \multicolumn{5}{|c|}{ Females } \\
\hline \multicolumn{5}{|l|}{ Black } \\
\hline 45-49 years & 454 & 1.4 & -0.4 & 3.3 \\
\hline 50-54 years & 734 & $3.1^{*}$ & 1.3 & 4.8 \\
\hline 55-59 years & 1,043 & $6.3^{*}$ & 4.7 & 7.9 \\
\hline 60-64 years & 1,386 & $6.0^{*}$ & 4.7 & 7.2 \\
\hline 65-69 years & 1,478 & $5.3^{*}$ & 3.7 & 6.9 \\
\hline 70-74 years & 1,353 & $3.6^{*}$ & 2.5 & 4.6 \\
\hline 75-79 years & 1,205 & $3.2^{*}$ & 2.2 & 4.3 \\
\hline 80-84 years & 899 & $3.4^{*}$ & 2.1 & 4.7 \\
\hline $85+$ years & 602 & $3.6^{*}$ & 1.3 & 5.9 \\
\hline
\end{tabular}

\section{Asian or Pacific Islander}

\begin{tabular}{|l|l|l|l|l|}
\hline 30-34 years & $\sim$ & $\sim$ & $\sim$ & $\sim$ \\
\hline 35-39 years & $\sim$ & $\sim$ & $\sim$ & $\sim$ \\
\hline 40-44 years & 110 & $3.0^{*}$ & 0 & 6 \\
\hline 45-49 years & 186 & $4.0^{*}$ & 0.7 & 7.4 \\
\hline 50-54 years & 286 & 2.8 & -1.1 & 6.9 \\
\hline 55-59 years & 474 & $5.6^{*}$ & 3.9 & 7.4 \\
\hline 60-64 years & 625 & $7.1^{*}$ & 5.5 & 8.7 \\
\hline $65-69$ years & 775 & $5.8^{*}$ & 4.7 & 7 \\
\hline $70-74$ years & 866 & $5.2^{*}$ & 3.8 & 6.6 \\
\hline $75-79$ years & 861 & $5.8^{*}$ & 4 & 7.7 \\
\hline $80-84$ years & 662 & $6.2^{*}$ & 4 & 8.5 \\
\hline $85+$ years & 475 & $9.7^{*}$ & 7.7 & 11.8 \\
\hline
\end{tabular}

\section{Hispanic}

\begin{tabular}{|l|l|l|l|l|}
\hline 30-34 years & $\sim$ & $\sim$ & $\sim$ & $\sim$ \\
\hline 35-39 years & 118 & $6.5^{*}$ & 2.7 & 10.5 \\
\hline 40-44 years & 206 & $6.2^{*}$ & 4 & 8.5 \\
\hline 45-49 years & 387 & $6.1^{*}$ & 3.9 & 8.3 \\
\hline 50-54 years & 563 & $6.4^{*}$ & 4.4 & 8.4 \\
\hline 55-59 years & 808 & $6.3^{*}$ & 4.3 & 8.3 \\
\hline 60-64 years & 1,035 & $7.2^{*}$ & 5.7 & 8.7 \\
\hline 65-69 years & 1,112 & $7.0^{*}$ & 5.5 & 8.6 \\
\hline 70-74 years & 1,133 & $4.9^{*}$ & 3.8 & 6 \\
\hline $75-79$ years & 1,080 & $4.7^{*}$ & 3.7 & 5.7 \\
\hline 80-84 years & 722 & $7.8^{*}$ & 6.6 & 9 \\
\hline $85+$ years & 515 & $6.8^{*}$ & 4.7 & 8.9 \\
\hline
\end{tabular}




\begin{tabular}{|c|c|c|c|c|}
\hline \multicolumn{5}{|l|}{ Males } \\
\hline Histological Types & Count & APC & LCI & UCI \\
\hline \multicolumn{5}{|l|}{ White } \\
\hline Adenocarcinoma, not otherwise specified & 39,237 & $3.3^{\star}$ & 3 & 3.5 \\
\hline Ductal adenocarcinoma excluding cystic or mucinous & 6,292 & $6.6^{*}$ & 6 & 7.3 \\
\hline $\begin{array}{l}\text { Ductal specified as arising from an intraductal } \\
\text { papillary mucinous neoplasm }\end{array}$ & 195 & $4.6^{\star}$ & 2 & 7.3 \\
\hline Non-secretory Endocrine & 2,572 & $3.8^{*}$ & 0.7 & 7.1 \\
\hline Secretory endocrine & 67 & 1.6 & -2.2 & 5.6 \\
\hline Carcinoid tumors & 1,244 & $32.5^{*}$ & 26.8 & 38.4 \\
\hline Acinar cell adenocarcinomas & 242 & $5.9 *$ & 3.1 & 8.7 \\
\hline Mucinous adenocarcinoma & 2,079 & $-3.0^{*}$ & -3.9 & -2.1 \\
\hline Other adenocarcinoma & 170 & -0.4 & -3 & 2.3 \\
\hline \multicolumn{5}{|l|}{ Black } \\
\hline Adenocarcinoma, not otherwise specified & 6,203 & $4.2^{*}$ & 3.7 & 4.7 \\
\hline Ductal adenocarcinoma excluding cystic or mucinous & 859 & $7.5^{\star}$ & 6.5 & 8.6 \\
\hline Endocrine: non-secretory & 328 & $6.1^{*}$ & 1.1 & 11.3 \\
\hline Mucinous adenocarcinoma & 274 & $-2.7^{\star}$ & -4.9 & -0.5 \\
\hline \multicolumn{5}{|l|}{ American Indian/Alaska Native } \\
\hline Adenocarcinoma, not otherwise specified & 281 & $7.0^{*}$ & 3.9 & 10.2 \\
\hline Ductal adenocarcinoma excluding cystic or mucinous & 40 & $13.4^{*}$ & 8.4 & 18.7 \\
\hline \multicolumn{5}{|l|}{ Asian or Pacific Islander } \\
\hline Adenocarcinoma, not otherwise specified & 3,334 & $5.7^{*}$ & 4.8 & 6.5 \\
\hline Ductal adenocarcinoma excluding cystic or mucinous & 649 & $8.5^{\star}$ & 6.6 & 10.4 \\
\hline Endocrine: non-secretory & 237 & $6.7^{\star}$ & 2 & 11.5 \\
\hline Mucinous adenocarcinoma & 183 & 2.1 & -1.3 & 5.5 \\
\hline \multicolumn{5}{|l|}{ Hispanics } \\
\hline Adenocarcinoma, not otherwise specified & 5,208 & $6.1^{*}$ & 5.4 & 6.7 \\
\hline Ductal adenocarcinoma excluding cystic or mucinous & 807 & $11.3^{*}$ & 9.6 & 13 \\
\hline Endocrine: non-secretory & 328 & $6.8^{*}$ & 2.9 & 10.8 \\
\hline Mucinous adenocarcinoma & 237 & 2 & -0.4 & 4.5 \\
\hline \multicolumn{5}{|l|}{ Females } \\
\hline \multicolumn{5}{|l|}{ White } \\
\hline Adenocarcinoma, not otherwise specified & 34,955 & $2.9 *$ & 2.6 & 3.1 \\
\hline Ductal adenocarcinoma excluding cystic or mucinous & 5,736 & $6.1^{*}$ & 5.5 & 6.8 \\
\hline Cystic adenocarcinoma & 185 & $-9.1^{*}$ & -12.1 & -6.1 \\
\hline $\begin{array}{l}\text { Ductal specified as arising from an intraductal } \\
\text { papillary mucinous neoplasm }\end{array}$ & 165 & $3.4^{*}$ & 0.9 & 5.9 \\
\hline Endocrine: non-secretory & 1,785 & $3.7^{\star}$ & 0.5 & 7 \\
\hline Secretory endocrine & 80 & -1.8 & -5 & 1.6 \\
\hline
\end{tabular}




\begin{tabular}{|c|c|c|c|c|}
\hline \multicolumn{5}{|l|}{ Females } \\
\hline \multicolumn{5}{|l|}{ White } \\
\hline Carcinoid tumors & 863 & $30.3^{*}$ & 24.5 & 36.4 \\
\hline Acinar cell adenocarcinomas & 82 & 2.1 & -1.3 & 5.7 \\
\hline Mucinous adenocarcinoma & 2,032 & $-2.7 *$ & -3.7 & -1.6 \\
\hline Other adenocarcinoma & 130 & -1.1 & -4.9 & 2.9 \\
\hline \multicolumn{5}{|l|}{ Black } \\
\hline Adenocarcinoma, not otherwise specified & 6,615 & $4.0^{*}$ & 3.5 & 4.4 \\
\hline Ductal adenocarcinoma excluding cystic or mucinous & 961 & $8.4^{*}$ & 6.8 & 10 \\
\hline Endocrine: non-secretory & 363 & 3.7 & -0.2 & 7.8 \\
\hline Carcinoid tumors & 228 & $26.9 *$ & 20.9 & 33.2 \\
\hline Mucinous adenocarcinoma & 355 & $-2.8^{*}$ & -4.7 & -0.9 \\
\hline \multicolumn{5}{|l|}{ American Indian/Alaska Native } \\
\hline Adenocarcinoma, not otherwise specified & 260 & 1.1 & -1.2 & 3.5 \\
\hline Ductal adenocarcinoma excluding cystic or mucinous & 43 & $7.1 *$ & 3.9 & 10.5 \\
\hline \multicolumn{5}{|l|}{ Asian or Pacific Islander } \\
\hline Adenocarcinoma, not otherwise specified & 3,552 & $5.7^{\star}$ & 5 & 6.4 \\
\hline Ductal adenocarcinoma excluding cystic or mucinous & 746 & $9.6^{*}$ & 7.5 & 11.7 \\
\hline Endocrine: non-secretory & 196 & 4.6 & -0.9 & 10.4 \\
\hline Mucinous adenocarcinoma & 195 & 0.1 & -3.2 & 3.5 \\
\hline \multicolumn{5}{|l|}{ Hispanic } \\
\hline Adenocarcinoma, not otherwise specified & 5,148 & $6.0 *$ & 5.3 & 6.7 \\
\hline Ductal adenocarcinoma excluding cystic or mucinous & 872 & $10.1^{*}$ & 8.2 & 12 \\
\hline Endocrine: non-secretory & 315 & $6.5^{\star}$ & 2.7 & 10.6 \\
\hline Mucinous adenocarcinoma & 307 & -0.4 & -2.5 & 1.7 \\
\hline \multicolumn{5}{|c|}{ APC = Annual percent change; UCI, LCI= Upper, Lower 95\% confidence interval. } \\
\hline \multicolumn{5}{|l|}{ Confidence intervals are $95 \%$ for trends. } \\
\hline \multicolumn{5}{|c|}{$\begin{array}{l}\text { Percent changes were calculated using } 1 \text { year for each end point; APCs were calculated using } \\
\text { weighted least squares method. }\end{array}$} \\
\hline \multicolumn{5}{|c|}{$\begin{array}{l}\text { Statistic could not be calculated due to lower number of cases or at least one year with no reported } \\
\text { cases. }\end{array}$} \\
\hline \multicolumn{5}{|l|}{ * The APC is significantly different from zero $(p<0.05)$} \\
\hline $\begin{array}{l}\text { Cases and annual percentage changes were only mentioned if } \\
\text { results after temporal trends analysis. }\end{array}$ & ere were & ough ca & es to yi & \\
\hline
\end{tabular}

Islander Hispanic (All Races) was lower as compared to the white(non-Hispanic) race (Table 4).

\section{Discussion}

Our statistical analysis for SEER 18 registry revealed reportable findings by histologic subtype and demographic details. Incidence rates of pancreatic carcinomas increased amongst white males and females consistently. Incidence rates for black, Asian, or pacific inlander and Hispanics all rose in recent years for both genders. Rates for American Indian/Alaskan natives were inconsistent throughout 2000-2017; however, the final annual percent change remained positive for males $(7.4 \%$, CI 4.7-10.1) and females (2.7\%, 0.7-4.7). Based on histological type, the overall increase in incidence remained secondary 

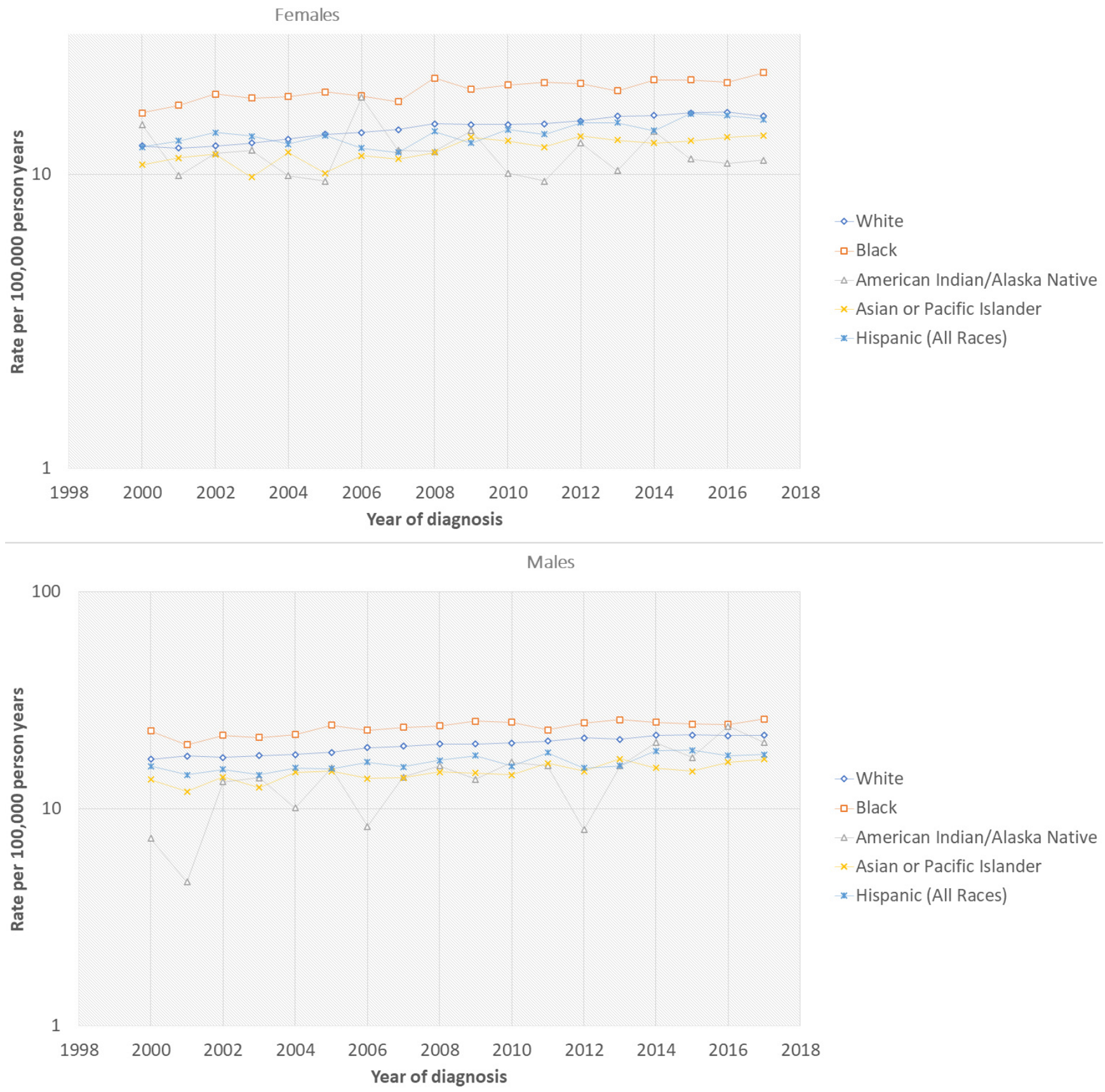

Figure 1. Pancreatic cancer incidence trends by gender and race based on SEER 18 (2000-2017). a: Females, b: Males.

to pancreatic adenocarcinoma, as mentioned in a previous similar study 5 .

Pancreatic carcinoma rates more predominantly rose in males when compared to females. In white males and females, there was a female prominence noted for incidence rates of age group 30-34 (Table 2, Table 3). Rates for other ethnicities for ages $<45$ were comparable or could not be analyzed due to the lower number of cases. Rates of common histologic types were more in males, except for cystic adenocarcinoma, secretory endocrine cancers, solid pseudopapillary cancers, and non-carcinomas. This differs based on the histological type could be attributed to cystic adenocarcinoma and solid pseudopapillary cancers as they had the highest female predominance. Previous research comparing male to female incidence rate ratios also came to a similar conclusion for long-term rates from 1975-2003 and $2000-2013^{5}$. A review of 718 patients in 2005 stated a female predominance of solid pseudopapillary pancreatic 

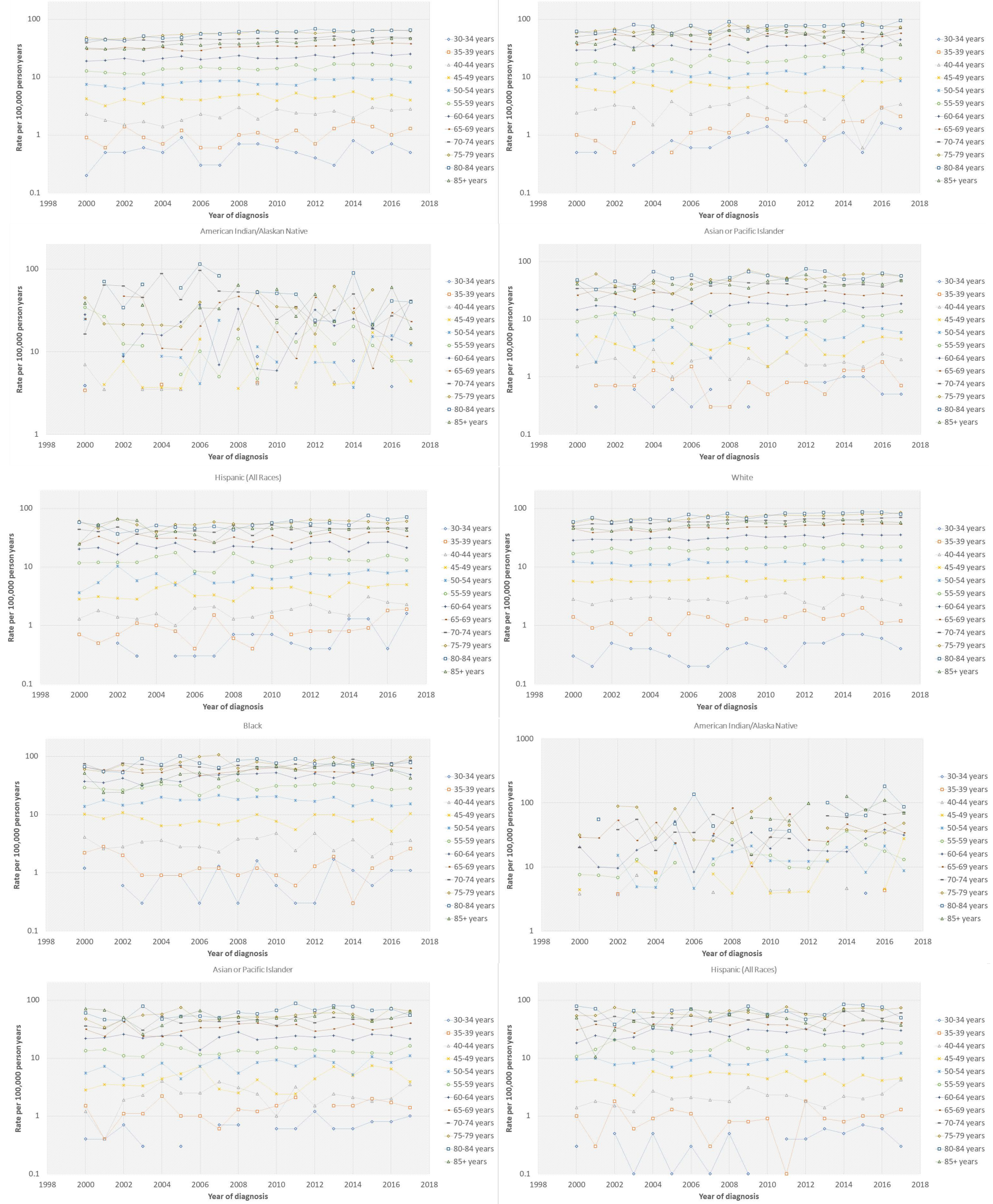

Figure 2. Pancreatic cancer incidence trends by age groups, gender and race based on SEER 18 (2000-2017). a: Males, b: Females. 

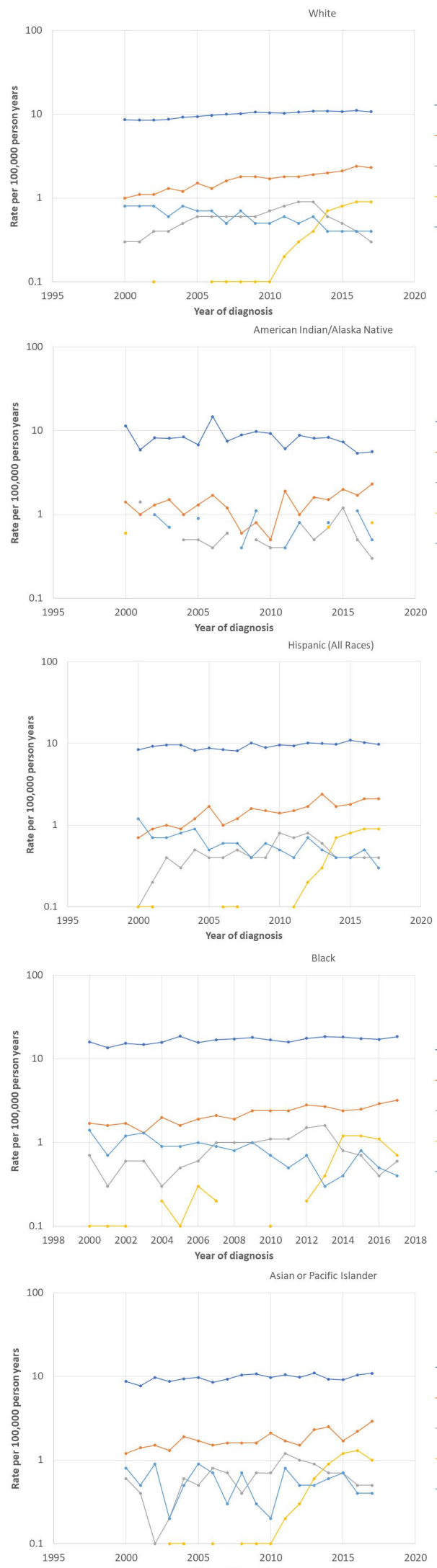

Black

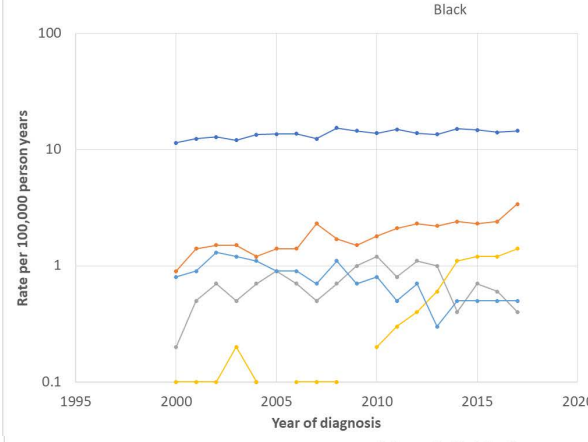

Asian or Pacific Islander
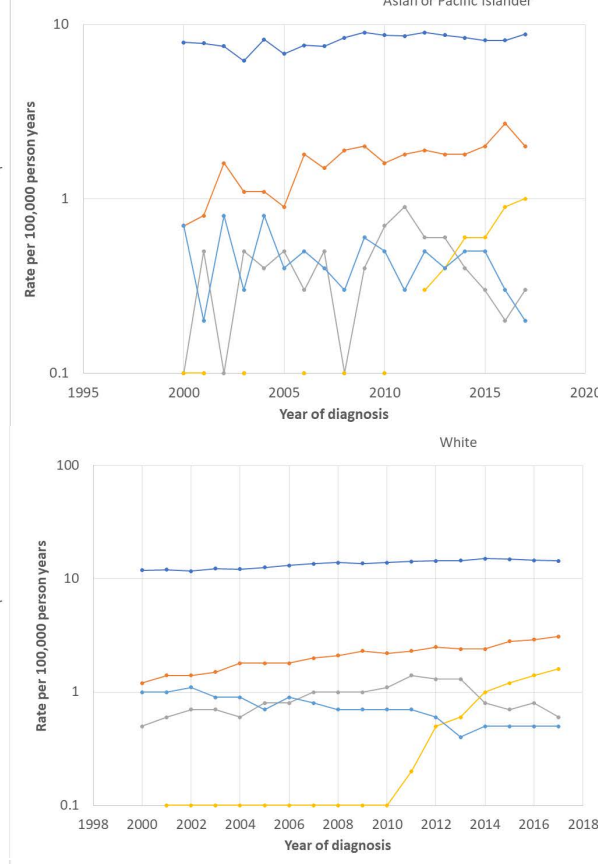

American Indian/Alaska Native
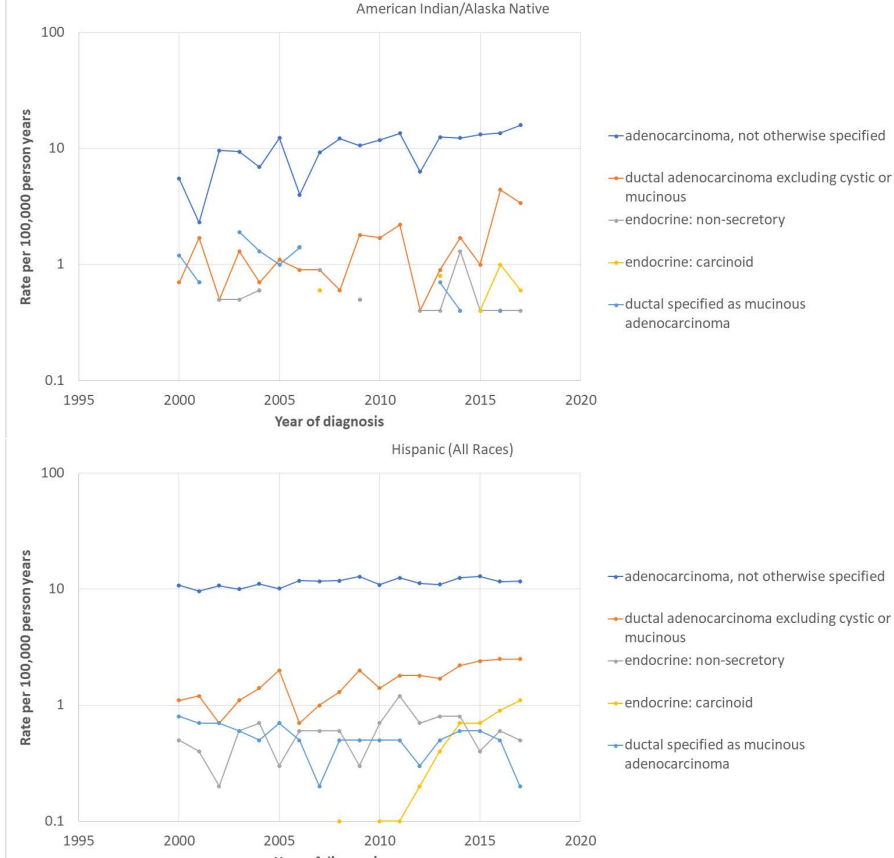

Figure 3. Pancreatic cancer incidence trends by age groups, gender and histological type based on SEER 18 (2000-2017). a: Males, b: Females. 
Table 3. Total cases, rates, and male to female incidence rate ratios, by age group and histologic type (2000-2017).

\begin{tabular}{|c|c|c|c|c|c|c|c|c|c|c|c|}
\hline \multicolumn{3}{|l|}{ Males and Females } & \multicolumn{3}{|c|}{ Males } & \multicolumn{3}{|c|}{ Females } & \multicolumn{3}{|c|}{ Male/Female } \\
\hline & Count & $\begin{array}{l}\% \text { of } \\
\text { total }\end{array}$ & Count & $\begin{array}{l}\% \text { of } \\
\text { total }\end{array}$ & Rate & Count & $\begin{array}{l}\% \text { of } \\
\text { total }\end{array}$ & Rate & IRR & LCI & UCI \\
\hline Total cases & 152548 & 100 & 79081 & 51.84 & 19.4 & 73467 & 48.15 & 14.7 & 1.32 & 1.30 & 1.33 \\
\hline \multicolumn{12}{|l|}{ Age groups } \\
\hline 30-34 years & 543 & 0.35 & 243 & 0.3 & 0.5 & 300 & 0.4 & 0.6 & 0.8 & 0.67 & 0.95 \\
\hline 35-39 years & 1,197 & 0.78 & 646 & 0.8 & 1.2 & 551 & 0.7 & 1 & 1.2 & 1.04 & 1.31 \\
\hline 40-44 years & 2,651 & 1.7 & 1,458 & 1.8 & 2.7 & 1,193 & 1.6 & 2.2 & 1.2 & 1.14 & 1.33 \\
\hline 45-49 years & 5,668 & 3.7 & 3,182 & 4.0 & 5.9 & 2,486 & 3.3 & 4.5 & 1.3 & 1.24 & 1.38 \\
\hline $50-54$ years & 10,364 & 6.7 & 6,041 & 7.6 & 11.9 & 4,323 & 5.8 & 8.2 & 1.5 & 1.39 & 1.50 \\
\hline 55-59 years & 15,815 & 10.3 & 9,025 & 11.4 & 20.4 & 6,790 & 9.2 & 14.5 & 1.4 & 1.37 & 1.45 \\
\hline 60-64 years & 20,378 & 13.3 & 11,465 & 14.49 & 32.5 & 8,913 & 12.1 & 23.1 & 1.4 & 1.37 & 1.44 \\
\hline 65-69 years & 23,226 & 15.2 & 12,645 & 15.98 & 46.8 & 10,581 & 14.4 & 34.4 & 1.4 & 1.32 & 1.39 \\
\hline 70-74 years & 23,319 & 15.2 & 12,082 & 15.27 & 60 & 11,237 & 15.3 & 46.1 & 1.3 & 1.27 & 1.34 \\
\hline 75-79 years & 21,789 & 14.2 & 10,418 & 13.17 & 69.4 & 11,371 & 15.5 & 56.9 & 1.2 & 1.19 & 1.25 \\
\hline 80-84 years & 16,467 & 10.7 & 7,476 & 9.4 & 72.5 & 8,991 & 12.2 & 57.4 & 1.3 & 1.23 & 1.30 \\
\hline $85+$ years & 11,131 & 7.2 & 4,400 & 5.5 & 53.8 & 6,731 & 9.1 & 40.5 & 1.3 & 1.28 & 1.38 \\
\hline \multicolumn{12}{|l|}{ Histological type } \\
\hline Total & 139267 & 100 & 72097 & 51.76 & 17.6 & 67170 & 48.23 & 13.5 & 1.31 & 1.29 & 1.32 \\
\hline Adenocarcinoma, not otherwise specified & 104,926 & 75.3 & 54344 & 75.4 & 13.3 & 50582 & 75.3 & 10.1 & 1.3 & 1.30 & 1.34 \\
\hline $\begin{array}{l}\text { Ductal adenocarcinoma excluding cystic } \\
\text { or mucinous }\end{array}$ & 17,026 & 12.2 & 8658 & 12 & 2.1 & 8368 & 12.5 & 1.7 & 1.25 & 1.21 & 1.3 \\
\hline Cystic adenocarcinoma & 408 & 0.2 & 118 & 0.1 & 0.0 & 290 & 0.4 & 0.1 & 0.5 & 0.4 & 0.6 \\
\hline $\begin{array}{l}\text { Ductal specified as arising from an } \\
\text { intraductal papillary mucinous neoplasm }\end{array}$ & 520 & 0.3 & 268 & 0.3 & 0.1 & 258 & 0.3 & 0.1 & 1.3 & 1.1 & 1.5 \\
\hline Endocrine: non-secretory & 6,167 & 4.4 & 3484 & 4.8 & 0.8 & 2683 & 3.9 & 0.6 & 1.5 & 1.4 & 1.6 \\
\hline Secretory endocrine & 203 & 0.1 & 94 & 0.1 & 0.0 & 109 & 0.1 & 0.0 & 0.9 & 0.9 & 1.3 \\
\hline Carcinoid tumors & 3,201 & 2.2 & 1753 & 2.4 & 0.4 & 1448 & 2.1 & 0.3 & 1.4 & 1.3 & 1.5 \\
\hline Acinar cell adenocarcinomas & 438 & 0.3 & 314 & 0.4 & 0.1 & 124 & 0.2 & 0.0 & 3.0 & 2.5 & 3.8 \\
\hline Mucinous adenocarcinoma & 5,696 & 4.1 & 2790 & 3.9 & 0.7 & 2906 & 4.3 & 0.6 & 1.2 & 1.1 & 1.2 \\
\hline Solid pseudopapillary tumors & 228 & 0.2 & 42 & 0.05 & 0.0 & 186 & 0.3 & 0.0 & 0.2 & 0.2 & 0.3 \\
\hline Other adenocarcinoma & 448 & 0.3 & 229 & 0.3 & 0.1 & 219 & 0.3 & 0.1 & 1.3 & 1.1 & 1.5 \\
\hline Other non-carcinomas & 6 & 0.004 & 3 & 0.004 & 0.0 & 3 & 0.004 & 0.0 & 0.9 & 0.1 & 7.7 \\
\hline
\end{tabular}

Rates are per 100000 person-years, age-adjusted to the 2000 US Standard Population (19 age groups - Census P25-1130).

IRR = Incidence Rate Ratios (based on unrounded rates); LCI, UCI = lower, upper 95\% confidence interval.

Confidence intervals (Tiwari mod) are 95\% for the ratios. 
Table 4. Total cases, rates, and male to female incidence rate ratios by gender and racial/ethnic group, (2000-2017).

\begin{tabular}{|c|c|c|c|c|c|c|c|c|c|c|}
\hline \multicolumn{6}{|c|}{ Males } & \multicolumn{5}{|c|}{ Females } \\
\hline Race & Count & Rate & IRR & LCI & UCI & Count & Rate & IRR & LCI & UCI \\
\hline White & 56,991 & 19.8 & 0 & 0 & 0 & 50,344 & 14.5 & 0 & 0 & 0 \\
\hline Black & 8,807 & 23.9 & 1.21 & 1.18 & 1.24 & 9,489 & 19.6 & 1.36 & 1.33 & 1.39 \\
\hline American Indian/Alaska Native & 396 & 14.9 & 0.75 & 0.67 & 0.84 & 383 & 11.8 & 0.82 & 0.74 & 0.91 \\
\hline Asian or Pacific Islander & 5,138 & 14.9 & 0.76 & 0.73 & 0.78 & 5,404 & 12.4 & 0.85 & 0.83 & 0.88 \\
\hline Hispanic (All Races) & 7,616 & 16.6 & 0.84 & 0.82 & 0.86 & 7,751 & 14.1 & 0.97 & 0.95 & 0.99 \\
\hline \multicolumn{11}{|c|}{ Rates are per 100000 person-years, age-adjusted to the 2000 US Standard Population (19 age groups - Census P25-1130). } \\
\hline \multicolumn{11}{|c|}{ IRR = Incidence Rate Ratios (based on unrounded rates); LCI, UCI = lower, upper 95\% confidence interval. } \\
\hline \multicolumn{11}{|c|}{ Confidence intervals (Tiwari mod) are 95\% for the ratios. } \\
\hline \multicolumn{11}{|c|}{ The reference group is White non-Hispanic. } \\
\hline
\end{tabular}

cancers; almost $90 \%$ occurred in younger females aged between 19 to 50 , with a ratio of close to $10: 1$ for females to males ${ }^{11}$. A systemic review also showed an increased predominance of cystic adenocarcinomas in females, and our results were consistent with these studies ${ }^{12}$.

An interesting finding in our analysis was the recent rise in pancreatic endocrine carcinoid tumors in all ethnicities. There was a male predominance with (IRR $1.4 \%$, CI 1.3-1.5). The rates were highest in white males and started to increase after 2010 in all races except American Indian/Alaska Native who showed sporadic rise (Figure 3). The black and Asian or pacific inlanders' incidence rates showed some plateau/decline in recent years (Figure 3). A previous study on pancreatic neuroendocrine tumors (PNETs) suggested that cigarette smoking, Type II Diabetes, and family history in a first-degree relative are independent risk factors for non-functional PNETs. Another independent risk factor for functional PNETs was found to be heavier consumption of alcohol ${ }^{13}$. No study currently comments solely on the risk factors of pancreatic carcinoid tumors, and it would be interesting to see associated risk factors for this recent rise. Our analysis revealed a rising rate of pancreatic carcinoid tumors with age, however, the results declined for ages $>75$ (results not shown).

Rates for intraductal papillary mucinous tumors and secretory endocrine carcinomas were similar in both genders. IPMN are malignancies of the pancreas that grow within the pancreatic ducts and produce mucin, hence the name. The risk factors of IPMN are not well understood. However, possible risk factors include insulin-dependent diabetes mellitus, chronic pancreatitis, positive family history, and smoking ${ }^{14}$. Our analysis revealed a decline in mucinous adenocarcinomas (Figure 3). This is consistent with the previously reported study. This can be explained by the fact that improved diagnostic imaging has enabled the detection of cystic or mucinous precursor lesions at an earlier stage. Our analysis only included only malignant lesions ${ }^{15}$.
Numerous risk factors exist for pancreatic carcinoma and could be the reason behind the rising incidence. Individuals with non-O blood groups (type $\mathrm{A}, \mathrm{AB}$, or $\mathrm{B}$ ) are more prone to the risk of pancreatic cancer ${ }^{16}$. A possible link between Helicobacter pylori infection and non-O blood type may also exist; however, evidence is still lacking ${ }^{17}$. Cystic fibrosis patients are at a greater risk of pancreatic cancer, especially adenocarcinoma, as their life span increases. It was also two to five-fold higher in patients with a history of organ transplantation ${ }^{18}$. Up to $10 \%$ of pancreatic cancer patients have a family history ${ }^{19}$. Some studies, though not explicit, identified a germline mutation involving a known susceptibility gene for pancreatic cancer (including BRCA, ATM, CDKN2A and PALB2) in patients with familial etiology $\mathrm{y}^{20}$.

Chronic pancreatitis is also a risk factor for pancreatic cancer ${ }^{21}$. This is hypothesized due to inflammation-induced metaplasia of the acinar cells into ductal cells by NF-kB and matrix metalloproteinases ${ }^{21}$. Patients with a type of neoplastic pancreatic cyst, intraductal papillary mucinous neoplasm of the pancreas (IPMN) can develop an invasive malignancy called IPMN-associated adenocarcinoma. These patients can also develop ductal adenocarcinoma $^{22}$. Smoking is the most common cause of pancreatic cancer. Studies show a linear relationship with the number of cigarettes smoked $^{23}$. However, the risk of pancreatic cancer decreases by $48 \%$ two years after smoking cessation. This is equivalent to that of non-smokers by 10 to 15 years after smoking cessation $^{23}$. Individuals who struggle with body weight develop pancreatic cancer at a relatively younger age with shorter survival compared to individuals with normal bodyweight ${ }^{24}$. Data regarding the effects of alcohol and coffee consumption has always been conflicting. Pooled analysis data showed a slight increase in the risk of pancreatic cancer in heavy drinkers and a high intake of coffee. This could be due to the confounder, which is cigarette smoking ${ }^{25}$. An inverse relationship between consumption of a healthy diet and pancreatic cancer ${ }^{26}$. However, the protective advantages of a healthy diet were only 
significant in men who were overweight/obese ${ }^{26}$. Low levels of selenium and lycopene were found in subjects who developed pancreatic cancer in later life, however, the results are unclear $^{27}$. Though studies have shown an association with Hepatitis $\mathrm{B}$ and $\mathrm{C}$ with pancreatic cancer, the magnitude of risk is still significantly less than hepatocellular cancer ${ }^{28}$. Numerous studies show an association between diabetes and pancreatic cancer. A meta-analysis showed the relative risk for pancreatic cancer in diabetes compared to the non-diabetic counterparts was $2.08^{29}$. Defects in metabolism of glucose, insulin resistance, and hyperinsulinemia are few etiologic factors for pancreatic cancer ${ }^{30-32}$. Increased risk of pancreatic cancer in patients with metabolic syndrome could be due to lower levels of adiponectin (a protein hormone linked to glucose level regulation and fatty acid breakdown), which results in a lack of insulin-sensitizing and anti-inflammatory capabilities ${ }^{33}$.

Our study had its limitations, especially from an etiological point of view, as SEER does not report any risk factors, which can influence these trends. Comorbidities like obesity, diabetes, smoking are also not reported. There is a concern of migrating patients in and out of areas registered in the SEER database and selection bias. The data of the SEER registry holds high-grade quality, and statistics based on population can be informative of risk patterns and trends based on time. The critical strength of our studies was the large sample size, as SEER 18 covers more recent cases up to 2017 and covers almost $30 \%$ of the US population. It also allowed us to analyze histological types of pancreatic cancer.

\section{Conclusion}

Pancreatic cancer incidence rates have continued to rise according to SEER 18 (2000-2017) in the USA. Our analysis concludes incidence of pancreatic cancer patterns based on demographical data and histologic type. We report the new rising incidence of pancreatic carcinoid tumors and the continued rise of pancreatic adenocarcinoma. We also report an increased incidence of cystic adenocarcinoma and solid pseudopapillary cancers in females as compared to males. These trends could be secondary to known risk factors for pancreatic carcinomas. Further comparative data regarding exclusive risk factors and contributing factors for histological subtypes of pancreatic carcinomas, specially PNETs, are required to understand the recent increase in incidence.

\section{Data availability}

All data underlying the results are available as part of the article and no additional source data are required.

\section{Author contributions}

Hassam Ali: Conceptualization, Methodology, Software, Validation, Writing- Reviewing and Editing. Meghana Vallabhaneni: Data curation, Writing- Original draft preparation. Rahul Pamarthy: Visualization, Project administration, Investigation. Shiza Sarfraz: Supervision, software, resources. Hadiqa Ali: Software, Validation. Hamza Rafique: Writing- Reviewing and Editing.
1. Bray F, Ferlay J, Soerjomataram I, et al.: Global cancer statistics 2018: GLOBOCAN estimates of incidence and mortality worldwide for 36 cancers in 185 countries. CA Cancer J Clin. 2018; 68(6): 394-424. PubMed Abstract | Publisher Full Text

2. Ward EM, Sherman RL, Henley SJ, et al:: Annual Report to the Nation on the Status of Cancer, Featuring Cancer in Men and Women Age 20-49 Years. I Natl Cancer Inst. 2019; 111(12): 1279-1297.

PubMed Abstract | Publisher Full Text | Free Full Text

3. Siegel RL, Miller KD, Jemal A: Cancer statistics, 2020. CA Cancer J Clin. 2020; 70(1): 7-30. PubMed Abstract | Publisher Full Text

4. Ilic M, Ilic I: Epidemiology of pancreatic cancer. World J Gastroenterol. 2016; 22(44): 9694-9705

PubMed Abstract | Publisher Full Text | Free Full Text

5. Gordon-Dseagu VL, Devesa SS, Goggins M, et al:: Pancreatic cancer incidence trends: evidence from the Surveillance, Epidemiology and End Results (SEER) population-based data. Int J Epidemiol. 2018; 47(2): 427-439. PubMed Abstract | Publisher Full Text | Free Full Text

6. Surveillance Research Program, NCIs Division of Cancer Control and Population Sciences: SEER Registries. (20 march 2021, date last accessed). Reference Source

7. Percy C, Van Holten V, Muir C: ICD- O International Classification of Diseases for Oncology. 2nd edn Geneva: World Health Organization, 1990. Reference Source

8. Fritz A, Percy C, Jack A, et al.: ICD-O International Classification of Diseases for Oncology. 3rd edn Geneva: World Health Organization, 2000. Reference Source

9. Surveillance Research Program, National Cancer Institute: SEER`Stat software. version 8.3.9.

Reference Source

10. Devesa SS, Donaldson J, Fears T: Graphical presentation of trends in rates. Am J Epidemiol. 1995; 141(4): 300-304. PubMed Abstract | Publisher Full Text

11. Papavramidis T, Papavramidis S: Solid pseudopapillary tumors of the pancreas: review of $\mathbf{7 1 8}$ patients reported in English literature. $\mathrm{Am}$ Coll Surg. 2005; 200(6): 965-972. PubMed Abstract | Publisher Full Text

12. Nilsson LN, Keane MG, Shamali A, et al.: Nature and management of pancreatic mucinous cystic neoplasm (MCN): A systematic review of the literature. Pancreatology. 2016; 16(6): 1028-1036. PubMed Abstract | Publisher Full Text

13. Ben Q, Zhong J, Fei J, et al.: Risk Factors for Sporadic Pancreatic Neuroendocrine Tumors: A Case-Control Study. Sci Rep. 2016; 6: 36073. PubMed Abstract | Publisher Full Text | Free Full Text

14. Fukushima N, Mukai K: Pancreatic neoplasms with abundant mucus production: emphasis on intraductal papillary-mucinous tumors and mucinous cystic tumors. Adv Anat Pathol. 1999; 6(2): 65-77. PubMed Abstract | Publisher Full Text

15. Kaur S, Baine MJ, Jain M, et al.: Early diagnosis of pancreatic cancer: challenges and new developments. Biomark Med. 2012; 6(5): 597-612. PubMed Abstract | Publisher Full Text | Free Full Text

16. Guo Z, Heydari AR, Wu W, et al.: Characterization of gene-specific DNA repair by primary cultures of rat hepatocytes. J Cell Physiol. 1998; 176(2): 314-322. PubMed Abstract | Publisher Full Text

17. Lowenfels $A B$, Maisonneuve $P$, Cavallini $G$, et al: Pancreatitis and the risk of pancreatic cancer. International Pancreatitis Study Group. N EnglJ Med. 
1993; 328(20): 1433-1437.

PubMed Abstract | Publisher Full Text

18. Maisonneuve $P$, Marshall $B C$, Lowenfels $A B$ : Risk of pancreatic cancer in patients with cystic fibrosis. Gut. 2007; 56(9): 1327-1328. PubMed Abstract | Publisher Full Text | Free Full Text

19. Olson SH, Kurtz RC: Epidemiology of pancreatic cancer and the role of family history. J Surg Oncol. 2013; 107(1): 1-7.

PubMed Abstract | Publisher Full Text | Free Full Text

20. Shindo K, Yu J, Suenaga M, et al.: Deleterious Germline Mutations in Patients With Apparently Sporadic Pancreatic Adenocarcinoma. J Clin Oncol. 2017; 35(30): 3382-3390.

PubMed Abstract | Publisher Full Text | Free Full Text

21. Liou GY, Döppler H, Necela B, et al.: Macrophage-secreted cytokines drive pancreatic acinar-to-ductal metaplasia through NF-KB and MMPs. / Cell Biol. 2013; 202(3): 563-577.

PubMed Abstract | Publisher Full Text | Free Full Text

22. Xiao SY: Intraductal papillary mucinous neoplasm of the pancreas: an update. Scientifica (Cairo). 2012; 2012: 893632.

PubMed Abstract | Publisher Full Text | Free Full Text

23. Bosetti C, Lucenteforte E, Silverman DT, et al.: Cigarette smoking and pancreatic cancer: an analysis from the International Pancreatic Cancer Case-Control Consortium (Panc4). Ann Oncol. 2012; 23(10): 1880-1888. PubMed Abstract | Publisher Full Text | Free Full Text

24. Li D, Morris JS, Liu J, et al.: Body mass index and risk, age of onset, and survival in patients with pancreatic cancer. JAMA. 2009; 301(24): 2553-2562. PubMed Abstract | Publisher Full Text | Free Full Text

25. Michaud DS, Vrieling A, Jiao L, et al:: Alcohol intake and pancreatic cancer: a pooled analysis from the pancreatic cancer cohort consortium (PanScan). Cancer Causes Control. 2010; 21(8): 1213-1225.

PubMed Abstract | Publisher Full Text | Free Full Text

26. Arem H, Reedy J, Sampson J, et al.: The Healthy Eating Index 2005 and risk for pancreatic cancer in the NIH-AARP study. J Nat/ Cancer Inst. 2013; 105(17): $1298-1305$.

PubMed Abstract | Publisher Full Text | Free Full Text

27. Burney PG, Comstock GW, Morris JS: Serologic precursors of cancer: serum micronutrients and the subsequent risk of pancreatic cancer. Am J Clin Nutr. 1989; 49(5): 895-900.

PubMed Abstract | Publisher Full Text

28. Huang J, Magnusson M, Törner A, et al.: Risk of pancreatic cancer among individuals with hepatitis $C$ or hepatitis $B$ virus infection: a nationwide study in Sweden. Br J Cancer. 2013; 109(11): 2917-2923. PubMed Abstract | Publisher Full Text | Free Full Text

29. Batabyal P, Vander Hoorn S, Christophi C, et al.: Association of diabetes mellitus and pancreatic adenocarcinoma: a meta-analysis of 88 studies. Ann Surg Oncol. 2014; 21(7): 2453-2462. PubMed Abstract | Publisher Full Text

30. Carreras-Torres R, Johansson M, Gaborieau V, et al.: The Role of Obesity, Type 2 Diabetes, and Metabolic Factors in Pancreatic Cancer: A Mendelian Randomization Study. J Natl Cancer Inst. 2017; 109(9): djx012.

PubMed Abstract | Publisher Full Text | Free Full Text

31. Michaud DS, Liu S, Giovannucci E, et al.: Dietary sugar, glycemic load, and pancreatic cancer risk in a prospective study. J Natl Cancer Inst. 2002; 94(17): 1293-1300.

PubMed Abstract | Publisher Full Text

32. Wolpin BM, Bao Y, Qian ZR, et al.: Hyperglycemia, insulin resistance, impaired pancreatic $\beta$-cell function, and risk of pancreatic cancer. J Nat Cancer Inst. 2013; 105(14): 1027-1035.

PubMed Abstract | Publisher Full Text | Free Full Text

33. Bao Y, Giovannucci EL, Kraft P, et al.: A prospective study of plasma adiponectin and pancreatic cancer risk in five US cohorts. J Natl Cancer Inst. 2013; 105(2): 95-103.

PubMed Abstract | Publisher Full Text | Free Full Text 


\section{Open Peer Review}

\section{Current Peer Review Status:}

\section{Version 1}

Reviewer Report 01 September 2021

https://doi.org/10.5256/f1000research.57873.r88966

(C) 2021 Sandhyavenu $\mathbf{H}$. This is an open access peer review report distributed under the terms of the Creative Commons Attribution License, which permits unrestricted use, distribution, and reproduction in any medium, provided the original work is properly cited.

\section{Harigopal Sandhyavenu}

Department of Cardiovascular Medicine, Mayo Clinic, Rochester, MN, USA

An interesting way of looking at the temporal trends of Pancreatic cancers. It is surprising to see increasing in the overall incidence, especially pancreatic endocrine carcinoid tumors among all ethnicities. Is it an actual rise in their number, or increasing positive tests with advanced diagnostic strategies? Either way this paper will allow the readers to look for possible correlation, if any. Overall this is an interesting article.

Is the work clearly and accurately presented and does it cite the current literature? Yes

Is the study design appropriate and is the work technically sound?

Yes

Are sufficient details of methods and analysis provided to allow replication by others? Yes

If applicable, is the statistical analysis and its interpretation appropriate? Yes

Are all the source data underlying the results available to ensure full reproducibility? Yes

Are the conclusions drawn adequately supported by the results? Yes

Competing Interests: No competing interests were disclosed.

Reviewer Expertise: General Internal Medicine, Cardiology, Geriatrics 


\section{I confirm that I have read this submission and believe that I have an appropriate level of expertise to confirm that it is of an acceptable scientific standard.}

Author Response 01 Sep 2021

\section{Hassam Ali}

Thank you for your review. For your question "Is it an actual rise in their number, or increasing positive tests with advanced diagnostic strategies?": Our reported cases are biopsy confirmed and malignant therefore an actual rise instead of incidental findings.

Competing Interests: No competing interests were disclosed.

Reviewer Report 12 July 2021

\section{https://doi.org/10.5256/f1000research.57873.r88964}

(C) 2021 Waqar S. This is an open access peer review report distributed under the terms of the Creative Commons Attribution License, which permits unrestricted use, distribution, and reproduction in any medium, provided the original work is properly cited.

\section{Syed Hamza Bin Waqar}

Department of Medicine, State University of New York, Downstate Health Sciences University, Brooklyn, NY, USA

Interesting article which is dealing with SEER database to study one of the most invasive pancreatic CA out there. I like the design of the study and it's a smart idea to build up a database from SEER. It is interesting to note that PNETs are now going up the trend, although the reason is uncertain. It might be something that scientists/doctors would like to look into moving forward as to why the incidence is increasing now? Is it just coincidence or is there is an actual correlation and if so, with what? A very thorough epidemiological, smart, and short study.

Is the work clearly and accurately presented and does it cite the current literature? Yes

Is the study design appropriate and is the work technically sound? Yes

Are sufficient details of methods and analysis provided to allow replication by others? Yes

If applicable, is the statistical analysis and its interpretation appropriate? Yes

Are all the source data underlying the results available to ensure full reproducibility? 
Yes

Are the conclusions drawn adequately supported by the results?

Yes

Competing Interests: No competing interests were disclosed.

Reviewer Expertise: Gastroenterology, Hematology-Oncology

I confirm that I have read this submission and believe that I have an appropriate level of expertise to confirm that it is of an acceptable scientific standard.

The benefits of publishing with F1000Research:

- Your article is published within days, with no editorial bias

- You can publish traditional articles, null/negative results, case reports, data notes and more

- The peer review process is transparent and collaborative

- Your article is indexed in PubMed after passing peer review

- Dedicated customer support at every stage

For pre-submission enquiries, contact research@f1000.com 